\title{
Running Multiple Instances of the Distributed Coordination Function for Air-Time Fairness in Multi-Rate WLANs
}

\author{
Mehmet Akif Yazici, Member, IEEE, and Nail Akar, Member, IEEE
}

\begin{abstract}
Conventional multi-rate IEEE 802.11 Wireless LANs (WLANs) are associated with the so-called performance anomaly to describe the phenomenon of high bit rate nodes being dragged down by slower nodes. This anomaly is known to be an impediment to obtaining high cumulative throughputs despite the employment of effective link adaptation mechanisms. To cope with the performance anomaly, air-time fairness has been proposed as an alternative to throughput fairness, the latter being a main characteristic of the IEEE 802.11 Distributed Coordination Function (DCF). In this paper, we propose a novel distributed air-time fair MAC (Medium Access Control) without having to change the operation of the conventional DCF. In the proposed MAC, each node in the system runs multiple instances of the conventional DCF back-off algorithm where the number of DCF instances for the nodes can be chosen in a distributed manner. Both analytical and simulation-based results are provided to validate the effectiveness of the proposed air-time fair MAC.
\end{abstract}

Index Terms-Wireless LAN, resource management, distributed algorithms.

\section{INTRODUCTION}

$\mathbf{T}$ HE IEEE 802.11 Working Group publishes the most widely deployed suite of protocols for Wireless Local Area Networks (WLAN). On the Medium Access Control (MAC) side, IEEE 802.11 employs a Carrier Sense Multiple Access with Collision Avoidance (CSMA/CA) MAC protocol with binary exponential back-off, known as Distributed Coordination Function (DCF) [1]. DCF defines a mandatory basic access mechanism and an optional Request-To-Send/ClearTo-Send (RTS/CTS) mechanism which is less often used in practice. The focus of this paper is on the basic access mechanism in which an 802.11 node with a frame to transmit listens to the channel first to detect an idle period of length at least equal to the Distributed Inter-Frame Space (DIFS). The node then sets its back-off timer value to an integer that is uniformly chosen in the interval $[0, C W-1]$, where $C W$ is set to $C W_{\min }$ (minimum contention window size) at the first transmission attempt. The back-off timer is then decremented at each slot as long as the channel is idle whereas it is stopped when a transmission is detected on the channel. Re-activation of the timer upon a transmission detection is done after the

Manuscript received February 9, 2013; revised August 27, 2013. The editor coordinating the review of this paper and approving it for publication was $\mathrm{P}$. Popovski.

The authors are with the Electrical and Electronics Engineering Dept., Bilkent University, Bilkent 06800, Ankara, Turkey (e-mail: \{yazici, akar\}@ee.bilkent.edu.tr).

Digital Object Identifier 10.1109/TCOMM.2013.111113.130120 channel is sensed idle after this transmission for at least a DIFS. The back-off timer hitting zero triggers the frame's first transmission. Once the destination host successfully receives the frame, it transmits an acknowledgment frame (ACK) after a short inter-frame space (SIFS) time. If the transmitting node does not receive an ACK within a specified ACK timeout for the transmitted frame, a collision is said to have taken place. Upon each collision, $C W$ is doubled until a maximum contention window size $C W_{\max }$ value is reached and the above back-off mechanism is repeatedly applied at each unsuccessful transmission. For more details, the reader is referred to [1].

Physical layer enhancements to the original 802.11 standard [1] made it possible to support raw data rates up to 54 Megabits per second (Mbps) [2],[3]. Despite the substantial increases in raw data rates for WLANs, since the used MAC (Medium Access Control) is the same, the actual throughput is much lower due to 802.11 overhead whose reduction is crucial for IEEE 802.11 standards to achieve higher throughputs [4]. Novel MAC-layer techniques besides PHY-layer enhancements have been explored in the IEEE 802.11n working group to reduce overhead so as to achieve a throughput surpassing $100 \mathrm{Mbps}$ [5]. Frame aggregation in which multiple frames are aggregated and transmitted at a single transmission opportunity as a burst is one such technique to reduce overhead [6].

IEEE 802.11 standards support multiple raw data rates and hence such networks are called multi-rate WLANs. As an example, the IEEE $802.11 \mathrm{~b}$ supports data rates in the set $\{1,2$, $5.5,11\}$ where the IEEE 802.11a standard supports data rates in the set $\{6,9,12,18,24,35,48,54\}$, all rates being in units of Mbps [2],[7]. Moreover, the 802.11 standards support link adaptation by which a host selects one of the available transmission rates at a given transmission opportunity based on channel conditions and/or application traffic type. Various link adaptation algorithms are developed to increase throughput and vendors use proprietary link adaptation algorithms [8]. Although link adaptation appears to be a powerful means to enhance throughput in multi-rate WLANs, its effective use in multi-user 802.11 WLANs has been shown to be limited [9]. To explain, consider a scenario of multiple hosts with a higher raw bit rate in addition to a single host with a lower bit rate as used in [9] with all frame sizes assumed to be the same. Since the CSMA/CA algorithm of DCF provides the same equal channel access probability to all hosts, the throughput of high rate hosts will be the same as the slow host. Therefore, DCF penalizes fast hosts and instead favors the slow host. 
This artifact is known as the performance anomaly problem of 802.11 DCF which impedes a direct relationship between the raw data rate and the actual throughput in scenarios with multiple users with different data rates [9]. Actually, DCF is throughput-fair when frame sizes used by different nodes are the same on the average. Time-based fairness is proposed in $[10],[11],[12]$ as an alternative to throughput fairness to cope with the performance anomaly problem. With time-based fairness, each competing node receives an equal share of the wireless channel occupancy time, i.e., air-time. A system achieving time-based fairness is called air-time fair. When air-time fair mechanisms are employed, the throughput of an individual node becomes strictly proportional with its raw bit rate and therefore high rate nodes will no longer be dragged down by slower ones, which leads to significantly higher cumulative throughputs [11]. A substantial amount of research has been dedicated to building air-time fair WLANs most of which focus on systems that require as minimal modification as possible to the existing widely deployed DCF.

In this paper, we propose a novel approach for achieving air-time fairness in IEEE 802.11 WLANs which is relatively simple to implement. In our proposed approach, a competing node runs multiple instances of the standard back-off algorithm at each node. Equivalently, a competing node behaves as a collection of multiple virtual nodes where each virtual node has its own DCF instant. When the back-off timer of a virtual node hits zero, then its controlling physical node decides to transmit the awaiting frame on behalf of the virtual node. Having multiple instances of the back-off algorithm at a given node increases the channel access probability when compared with ordinary nodes with a single DCF. We propose to use the multiplicity of back-off algorithm instances as an instrument to deliver air-time fairness. Consider a competing node $i$ that runs $N_{i}$ instances of the basic back-off algorithm. Let us assume that each node $i$ requires an average airtime $E\left[A_{i}\right]$ at each of its transmission opportunities. Let $A_{\max } \geq E\left[A_{j}\right], \forall j$ be a value known to all nodes. The particular choices for $A_{\max }$ will later be discussed in the sequel. We propose that the parameter $N_{i}$ is set to $N_{i}=\frac{A_{\max }}{E\left[A_{i}\right]}$ which can be done in a distributed manner once all nodes agree on $A_{\max }$. Note that the parameter $N_{i}$ need not be an integer in which case we propose a novel distributed mechanism that appropriately switches between $N_{i-}=\left\lfloor N_{i}\right\rfloor$ and $N_{i+}=\left\lceil N_{i}\right\rceil$ back-off algorithms. A novel Markov chain-based analytical model is provided in the current paper for the validation of this switching mechanism. Through extensive simulations, we show that the method we propose achieves air-time fairness at the expense of an acceptable reduction in channel utilization. The proposed method can also be used in conjunction with frame aggregation to substantially mitigate this utilization reduction which will be described in the sequel.

The organization of the paper is as follows. In Section 2, we give an overview of related work on air-time fairness. Section 3 addresses the proposed method and the stochastic model used to analyse air-time fairness. Section 4 addresses a number of practical aspects concerning the proposed method. In Section 5, we present our simulation results to demonstrate the effectiveness of the proposed approach. Finally, we conclude.

\section{RELATED WORK}

In this section, we overview related work on existing approaches that promise to deliver air-time fairness in WLANs. The first approach is based on the use of contention window parameter $C W_{\min }$ as an instrument to achieve air-time fairness. The references [13] and [14] analytically show for DCF that under certain assumptions, the nodal throughput is inversely proportional with the $C W_{\min }$ value of the node. In particular, air-time fairness can be achieved if the initial contention window size $C W_{\min }$ is chosen to be inversely proportional with the raw bit rate. Using $C W_{\min }$ adjustment for more general service differentiation purposes has also appeared in [15],[16],[17]. In [18], an algorithm for selecting optimal $C W_{\min }$ values is proposed. This reference also explores the usage of the Arbitration Interframe Space (AIFS) value defined in IEEE 802.11e for air-time usage control. The disadvantage of the method given in this study is that it requires recomputation whenever a station joins or leaves the network, or changes its rate. A neural network-based solution for finding $C W_{\min }$ and AIFS values to achieve airtime fairness is proposed in [19]. The main advantage of the $C W_{\text {min }}$-approach to deliver air-time fairness is in its simplicity of implementation and the preservation of the DCF mechanism. Several drawbacks of this approach within the scope of air-time based fairness are given below:

- The relationship between $C W_{\min }$ and the nodal throughput is valid only for regimes where the collision probabilities are small. Actually, the relationship between $C W_{\min }$ and the nodal throughput is sensitive to system parameters such as number of nodes, choice of initial congestion windows, etc. For example, a simulation study of [15] demonstrates that the throughput ratio between two classes of nodes with a fixed $C W_{\min }$ ratio is slightly sensitive to the number of nodes in each class. Similar results also appear in [18].

- Large initial contention windows are necessary when the ratio between the lowest and highest raw bit rates is relatively large. This can lead to a considerable underutilization of the channel [20].

- In actual implementations, $C W_{\min }$ needs to be a power of two [16]. Therefore, perfect air-time fairness between two nodes can not be achieved if the raw bit rate ratio is not a power of two.

In order to attack the long contention window sizes problem, in [20], the authors propose an on-line extension of the 802.11 DCF that dynamically adapts the minimum contention window of contending stations to achieve air-time fairness. However, each node is assumed to be aware of the number of competing nodes in the network which is difficult to manage in a distributed way. In [21], the authors propose a modification to the original CSMA/CA algorithm in which the contention windows of contending nodes are adjusted based on an estimator of the number of idle slots and the authors demonstrate high cumulative throughput as well as improved time-based fairness relative to the DCF. Despite the merits demonstrated in [21], deviation from the widely accepted CSMA/CA appears to be a drawback. 
Packet fragmentation is another approach to achieve air-time fairness. The reference [22] proposes a solution where packets from higher layers are fragmented based on the raw bit rate. In this solution, nodes with high bit rates use a frame size equal to the MTU (maximum transmission unit) whereas slow nodes fragment their packets so as to transmit smaller frames at each transmission opportunity. A similar cross-layer scheme is proposed in [23] that uses IP path MTU discovery so as reduce the number of bytes per frame sent by lower bit rate nodes while allowing higher bit rate nodes to send full size frames. An immediate drawback of the fragmentation-based approach is an increase in overhead due to fragmentation especially when most nodes are slow. Implementation complexity is another drawback due to need for cross-layer interaction.

Another category of solutions is the frame aggregation approach which is proposed in the IEEE 802.11e standard in which a transmission opportunity (TXOP), also referred to as the maximum channel occupation time, is broadcasted by the base station to each contending node. Consequently, nodes can aggregate their awaiting frames for transmission as long as the channel occupancy time does not exceed TXOP. Frame aggregation is also a crucial component of $802.11 \mathrm{n}$ due to the benefits it offers due to the significant reduction of overhead [5]. Frame aggregation can be used as a means of achieving air-time fairness and nodes with better channel conditions are allowed to send multiple frames at a transmission opportunity as opposed to low bit rate nodes that do not perform aggregation. The reference [24] proposes a dynamic and distributed aggregation mechanism which addresses the performance anomaly in both UDP and TCP scenarios by achieving time-based fairness in nearly all of the tested configurations. There are also existing results on optimal aggregation policies in $802.11 \mathrm{n}$ that can substantially increase aggregate throughput [25]. The reference [26] formulates DCF with respect to mixed data rates and packet sizes, and offers an adaptive packet size adjustment method. The reference [27] demonstrates the advantages of TXOP operations over the legacy 802.11 DCF and compare different TXOP managing policies in order to obtain the optimal one. Although TXOP can be used as an effective means of providing air-time fairness, the following drawbacks are identified:

- Frames are typically of variable size and further mechanisms including fragmentation are needed to transmit a number of frames within TXOP.

- Frame aggregation is generally used as a means of reducing overhead and thus enhancing cumulative throughput. If this method is used for air-time fairness, then slow nodes would not benefit from aggregation as much in case they dominate the user type.

- Let us assume all frames to be of the same length for the sake of simplicity. In the TXOP approach to deliver airtime fairness, the TXOP may be defined to be the time required for the slowest node to transmit a single frame. Let us now assume a $802.11 \mathrm{~b}$ WLAN occupied by two nodes with $11 \mathrm{Mbps}$ raw bit rates. In this case, when a node has channel access, it will transmit 11 back-toback frames. Clearly, such a scheme presents unfairness between these two nodes in the short term. The situation worsens when the ratio between the lowest and highest raw bit rates is even larger.

- Frame aggregation may lead to relatively poor delay performance as shown in [19].

A discrete-time Markov model for the performance analysis of the Enhanced Distributed Channel Access (EDCA) function of the IEEE 802.11e standard with EDCA parameters including $C W_{\min }$, TXOP, and AIFS is presented in [28]. The references [29], [30], and [31] offer cross-layer solutions that adjust the sending rates of the stations at the transport or application layers. The drawback of these solutions basically lies in their cross-layer design. The references [32] and [33] study the unfairness problem between uplink and downlink flows whereas [34] investigates fairness in terms of throughput and packet delays among users with diverse channel conditions due to the mobility and fading effects in WLANs.

\section{Proposed MAC and Its Analytical Modeling}

Let us assume a WLAN with $K$ users (nodes, stations). We assume saturated users which always have frames to send and we also assume that link adaptation at each node $i, 1 \leq i \leq K$, is done at each node in a way that frame error probabilities can be neglected. The case of non-zero frame error probabilities are left for future research. In order to include the possibility of frame aggregation, we define a burst to be a number of back-to-back frames to be transmitted at given transmission opportunity. A burst may correspond to a single frame if frame aggregation is not allowed. Fig. 1 illustrates a snapshot of the air-time utilization of the WLAN channel in time which consists of alternating idle and busy periods. When the user $i$ transmits successfully, i.e., no collisions take place, the channel is said to be occupied for an air-time of $A_{i}$ with mean $E\left[A_{i}\right]$. Actually, $A_{i}=B_{i} / R_{i}$ where $B_{i}$ is the burst size and $R_{i}$ is the raw bit rate, of user $i$. When a transmission occurs, this transmission belongs to user $i$ with probability $p_{i}$. Transmissions are followed with idle periods whose duration is a random variable denoted by $A_{I}$ with mean $E\left[A_{I}\right]$. In this generic model, there is flexibility in what constitutes an idle or busy period. If air-time fairness is sought only in terms of air-time required for the transmission of payloads, all header transmissions at the higher and MAC layers may be counted towards the idle period. The random variable $A_{I}$ has a fixed part which is examined in detail in [9] but also has a varying component stemming from contention periods which may dominate with increasing number of users $K$. The modeling and analysis of the idle period $A_{I}$ has received a lot of attention in the literature [35],[9]. In this paper, we will not attempt to obtain a stochastic model for $A_{I}$ but rather focus on the air-time utilization values of individual users which are crucial for air-time fairness. Note that users with low bit rates tend to occupy the WLAN channel longer at each transmission opportunity. $A_{i}$ can also vary for the same user $i$ in time due to varying frame lengths but we assume that $E\left[A_{i}\right]$ is known to user $i$.

Let $U_{i}$ denote the air-time utilization of user $i$ which is defined as the overall air-time consumed by successful transmissions of user $i$ over a large time window divided by 


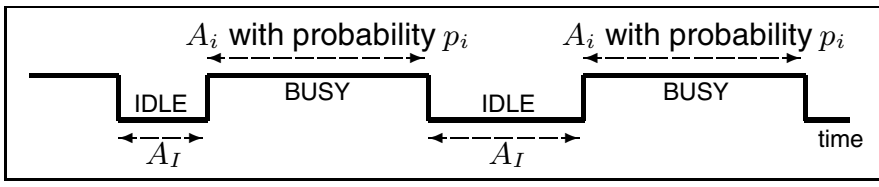

Fig. 1. A snapshot of the air-time utilization of a random access WLAN

the window length. With this definition,

$$
U_{i}=\frac{p_{i} E\left[A_{i}\right]}{E\left[A_{I}\right]+\sum_{j=1}^{K} p_{j} E\left[A_{j}\right]} .
$$

The overall channel utilization $U$ is then given by $U=$ $\sum_{j=1}^{K} U_{j}$. If $U_{i}=U_{j}, i \neq j, \forall i, j$ then we say the system is air-time fair. The IEEE $802.11 \mathrm{DCF}$ is known to provide equal channel access probabilities, i.e., $p_{i}^{(D C F)}=1 / K$, for all $i$. Denoting the air-time utilization of user $i$ in DCF by $U_{i}^{(D C F)}$, we have

$$
U_{i}^{(D C F)}=\frac{E\left[A_{i}\right]}{K E\left[A_{I}\right]+\sum_{j=1}^{K} E\left[A_{j}\right]} .
$$

It is therefore clear that air-time utilizations of users may be different with DCF due to either different raw bit rates or frame lengths. One possibility is to control $E\left[A_{i}\right]$ by frame aggregation in a way that $E\left[A_{i}\right]=E\left[A_{j}\right], i \neq j, \forall i, j$. However, the scope of the current paper is on an alternative approach by controlling $p_{i}$ which requires a modification to the DCF. In this article, we assume that the user $i$ runs $N_{i}$ instances of the DCF where $N_{i}$ is set to $\frac{A_{\max }}{E\left[A_{i}\right]}$ where the value $A_{\max }$ is such that $A_{\max } \geq E\left[A_{j}\right], \forall j$, and is known to each user. In the current paper, we propose that $A_{\max }$ is set to the time to transmit the largest possible frame with the minimum data rate the protocol supports, which then lends itself to a distributed implementation. With this choice of $A_{\max }, N_{i} \geq 1$ for all $i$ but $N_{i}$ need not be an integer for some user $i$. Let us first assume that $N_{i}$ is an integer for all $i, 1 \leq i \leq K$ in which case user $i$ behaves as a collection of $N_{i}$ virtual users each running its own DCF. We call this architecture Multiple DCF (MDCF). Consequently, the channel access probability for user $i$ for MDCF denoted by $p_{i}^{(M D C F)}$ can be written as

$$
p_{i}^{(M D C F)}=\frac{N_{i}}{\sum_{j=1}^{K} N_{j}}=\frac{1}{E\left[A_{i}\right] \sum_{j=1}^{K}\left(1 / E\left[A_{j}\right]\right)} .
$$

Finally, the air-time utilization of user $i$ in MDCF denoted by $U_{i}^{(M D C F)}$ is written as

$$
U_{i}^{(M D C F)}=\frac{1}{K+E\left[A_{I}\right] \sum_{j=1}^{K}\left(1 / E\left[A_{j}\right]\right)} .
$$

Since, the right hand side of (4) does not depend on $i, U_{i}$ is independent of $i$ and consequently, MDCF is air-time fair for integer $N_{i}$.

In the case of non-integer $N$ values, one can attack this problem by scaling up $A_{\max }$. As an example, assume $K=2$, $N_{1}=1.4$ and $N_{2}=3.1$. One can achieve air-time fairness by running 14 (31) instances of the DCF for user 1 (2) but at the expense of lowered cumulative throughputs stemming from significant increases in the overall number of DCFs in the system and therefore $E\left[A_{I}\right]$. We will next study an alldistributed approach still based on MDCF in the case of noninteger $N_{i}$. For the sake of simplicity, consider the particular case $K=2, N_{2}$ is an integer but $N_{1}$ is not. Let $N_{1-}=\left\lfloor N_{1}\right\rfloor$ and $N_{1+}=\left\lceil N_{1}\right\rceil$. We propose a MAC for user 1 as follows. User 1 runs $N_{1-}$ instances of the DCF for a geometrically distributed period of mean $B_{1-}$ transmissions belonging to user 1 and then switches to $N_{1+}$ instances for again a geometrically distributed period of mean $B_{1+}$ of its own transmissions. For the sake of convenience, we set $B=B_{1-}+B_{1+}$ and $B_{1-}=a_{1} B, B_{1+}=\left(1-a_{1}\right) B=b_{1} B$. Another way of describing this operation is as follows. Assume that user 1 is running $N_{1-}$ instances of the DCF. When user 1 completes a successful transmission, a new instance of the DCF is added with probability $\frac{1}{a_{1} B}$. Similarly, when user 1 is running $N_{1+}$ instances of the DCF and when it completes a successful transmission, one of the existing DCF instances is dropped with probability $\frac{1}{b_{1} B}$. Note that $B$ can be chosen large enough to ensure that these two values correspond to probabilities. Let $T_{i}, i=1,2$ denote the total air-time consumed by user $i$ over a period of $B$ successful transmissions of user 1. It is obvious that $T_{1}=B E\left[A_{1}\right]=B A_{\max } / N_{1}$. It is not difficult to write

$$
T_{2}=\frac{A_{\max }}{N_{2}}\left(\frac{N_{2}}{N_{1-}} a_{1} B+\frac{N_{2}}{N_{1+}}\left(1-a_{1}\right) B\right) .
$$

In order to ensure air-time fairness, we should have $T_{1}=T_{2}$ which then yields an equation in the unknown $a_{1}$. Solving for $a_{1}$, we have

$a_{1}=\frac{N_{1-}}{N_{1}}\left(N_{1+}-N_{1}\right), \quad b_{1}=1-a_{1}=\frac{N_{1+}}{N_{1}}\left(N_{1}-N_{1-}\right)$.

Since the choice of $a_{1}$ or $b_{1}$ does not depend on any parameters of user 2, the alternating MDCF policy proposed for user 1 can be implemented in an all-distributed fashion. The choice of $B$ can have some implications. If $B$ is chosen to be very large, it is clear that short-term deviation from air-time fairness can result. On the other hand, if $B$ is chosen to be small, then transient effects stemming from high frequency insertions and deletions of DCF instances may lead to undesirable behavior.

Although we have shown air-time fairness for $K=2$ and for only integer $N_{2}$, the choice of the switching parameter $a_{i}$ for user $i, 1 \leq i \leq K$ :

$$
a_{i}=\frac{N_{i-}}{N_{i}}\left(N_{i+}-N_{i}\right), \quad b_{i}=1-a_{i}=\frac{N_{i+}}{N_{i}}\left(N_{i}-N_{i-}\right),
$$

as a generalization of (5) also leads to air-time fairness for arbitrary $K$ and non-integer $N_{i}, 1 \leq i \leq K$ where $N_{i-}=\left\lfloor N_{i}\right\rfloor$ and $N_{i+}=\left\lceil N_{i}\right\rceil$. Actually, it is true for MDCF that the identity (3) holds despite non-integer $N_{j}$ and the expression for $U_{i}^{(M D C F)}$ in (4) given for integer $N_{i}$ remains intact for non-integer $N_{i}$ as well, provided that each user $i$ switches among $N_{i-}$ and $N_{i+}$ instances according to (6). For the sake of convenience, the proof of the above statement is given in the Appendix. This leads us to the conclusion that air-time fairness is achievable in an all-distributed manner for an arbitrary number of users in the WLAN.

Let us assume that a user running $N$ instances of the DCF, has a frame to transmit. As an example, let $N=3$ and $C W_{\min }=32$. Let the initial backoff timer values of the 
three DCF instances be 3,12, and 24. Then after DIFS plus three slots (assuming an idle channel), the node will transmit on behalf of the first virtual node. If the transmission queue is empty at the epoch of reception of the acknowledgment of this frame, then all contention windows are reset. Upon an arriving frame, contention windows of all three nodes will (independently) be initialized according to DCF rules. Otherwise, the initial contention window of the first virtual node is chosen uniformly from the set $\left\{0, \ldots, C W_{\text {min }}-1\right\}$ and the other two will continue to be decremented at each slot as long as the channel is idle. An interesting situation arises when the backoff timer values of multiple virtual nodes turn out to be identical. As an example, let the initial backoff timer values of the three DCF instances be 12,12, and 24. If this were a real WLAN, there would have been a collision due to the two virtual nodes. However, since the node associated with these virtual nodes will realize that this is an internal collision, it defers from transmitting. We call this behavior Internal Collision Prevention (ICP). We propose that the virtual nodes causing the internal collision behave like they experienced a real collision, i.e., their contention windows are doubled. Note that ICP behavior deviates from the mathematical model previously described in order to avoid further reduction in channel utilization, and thus the cumulative throughput. However, we show through simulations that ICP has marginal impact on airtime fairness but is obviously beneficiary in terms of overall channel utilization. In all the numerical examples to follow, we employ ICP.

\section{Practical Aspects}

In this section, we offer solutions to two issues in practical implementations for MDCF. The first issue is assigning a value to $A_{\max }$ which needs to be larger than $\max _{j} E\left[A_{j}\right]$. The ideal value would be $A_{\max }=\max _{j} E\left[A_{j}\right]$ but this would in general require communication between stations to find out and disseminate this value. Instead, we propose to set $A_{\max }$ to the ratio of the maximum transmission unit allowed to the minimum data rate supported. Then, each station can independently calculate $A_{\max }$ since the maximum transmission unit and the minimum supported data rate are known through protocol parameters.

Secondly, we assert that a station knows the mean of the airtime it requires, $E\left[A_{j}\right]$. To this end, we propose that a station can compute the mean payload it transfers at each transmission opportunity using damped averaging, and calculate $E\left[A_{j}\right]$ by dividing this value by its current data rate. Specifically, starting with the size of a maximum transmission unit as the initial value, a station $j$ computes its current payload estimate, $B_{j}^{e}(n)$ using its previous estimate, $B_{j}^{e}(n-1)$ via

$$
B_{j}^{e}(n)=\alpha B_{j}^{e}(n-1)+(1-\alpha) B_{j}(n)
$$

after each successful transmission, where $B_{j}(n)$ is the size of the transmitted payload.

\section{Simulation Study}

The simulation study is carried out via an event-based simulator using Matlab. The inter-frame spaces and the ACK mechanism including the ACK timeout are implemented, but
TABLE I

SIMULATION PARAMETERS

\begin{tabular}{lrlr}
\hline Slot time & $20 \mu \mathrm{s}$ & $C W_{\min }$ & 32 \\
DIFS period & $50 \mu \mathrm{s}$ & $C W_{\min }^{(M D C F)}$ & 156 \\
SIFS period & $10 \mu \mathrm{s}$ & $C W_{\max }$ & 1024 \\
ACK timeout & $300 \mu \mathrm{s}$ & $C W_{\max }^{(M D C F)}$ & 4992 \\
ACK frame & 14 bytes & Max. frame size & 1500 bytes \\
\hline
\end{tabular}

propagation delays are ignored. We also assume there are no hidden nodes. We define the air-time fairness metric $A F$ in a $K$-node scenario as the ratio of the minimum of the air-times that the nodes get, to their maximum:

$$
A F=\min _{1 \leq i \leq K} U_{i} / \max _{1 \leq i \leq K} U_{i} .
$$

The parameter $A F$ varies between 0 (worst case) and 1 (ideal air-time fairness). We present six numerical examples by which we investigate the performance of MDCF with the help of three metrics: (i) air-time fairness $A F$, (ii) channel utilization $U$, and (iii) cumulative throughput $T$ which is defined as the long-term average rate of successful bits transmitted through the channel. We experimented using IEEE $802.11 \mathrm{~b}$ parameters which are given in Table I.

Another important point with MDCF is that it yields a system with more participants compared with the standard DCF. The optimal value of $C W_{\min }$ is shown in [36] to depend linearly on the number of stations. Therefore, the value of $C W_{\min }$ for DCF should be multiplied with the expected increase in the number of virtual nodes with MDCF. For the specific case of IEEE $802.11 \mathrm{~b}$, the mean increase in the number of (virtual) nodes with MDCF relative to DCF is $(1+2+5.5+11) / 4=4.875$ in case we have an equal number of users at each raw bit rate. Therefore, we suggest that the $C W_{\min }$ value for MDCF, denoted by $C W_{\min }^{(M D C F)}$, to be set to $156=32 \times 4.875$ (unless stated otherwise) as opposed to 32 which is the default value of $C W_{\min }$ for the standard DCF in typical implementations. Throughout the article, we set $B=100$. Experimentation with varying $B$ reveal that unless $B$ is chosen small (order of ones) or large (order of several thousands), the choice of the parameter $B$ has marginal effect on MDCF performance. Let us assume that either the value $\frac{1}{a_{i} B}$ or $\frac{1}{b_{i} B}$ does not correspond to a probability for this choice of $B$ for some user $i$. One can then increase $B$ to solve the problem. However, this also means that $a_{i}$ or $b_{i}$ would be relatively small. Consequently, the number $N_{i}$ should be very close to either $N_{i-}$ or $N_{i+}$; see identity (6). Therefore, in this case, switching would not be necessary at all which is the approach we take in this paper by fixing $B$ to 100 . We ran all the simulations until all virtual nodes have successfully transmitted at least 10000 frames except for Example IV which has a fixed simulation time. The parameter $A_{\max }=12 \mathrm{~ms}$ which is the air-time required to transmit a $1500 \mathrm{~B}$ frame at $1 \mathrm{Mbps}$.

Example I: In the first example, a simulation study is carried out for a scenario with two nodes whose data rates are varied in the range $1 \mathrm{Mbps}$ up to $11 \mathrm{Mbps}$ in $0.5 \mathrm{Mbps}$ steps. Obviously, this set of data rates includes non-standard values for the purpose of justifying the air-time fairness feature of MDCF. We allow one frame transmission at a given 


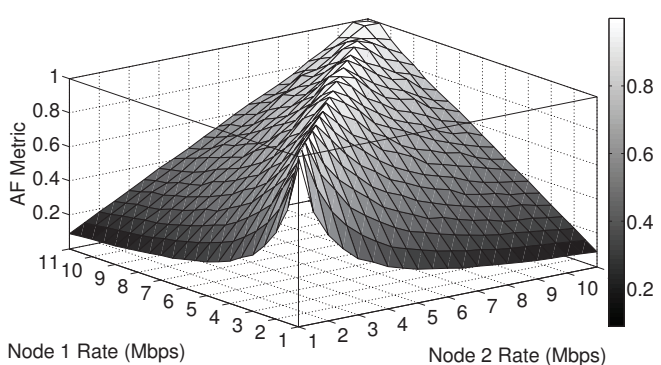

(a) Standard DCF

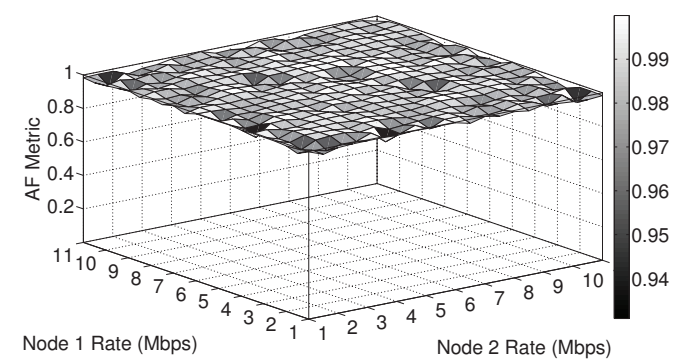

(b) MDCF

Fig. 2. Air-time fairness comparison of standard DCF and MDCF as a function of raw bit rates.

transmission opportunity and the frame length is fixed to 1500 bytes for all users. The results are given in figures 2, 3 and 4. It is observed that MDCF achieves almost perfect air-time fairness whereas the air-time fairness metric of the standard DCF is proportional with the ratio of the data rates. Also, a substantial gain in the cumulative throughput is demonstrated especially for asymmetric data rates which is an immediate consequence of air-time fairness. Note that the price of the air-time fairness in terms of channel utilization reduction is no more than $8 \%$ for MDCF. Standard DCF suffers a reduction in channel utilization only due to the increase in the rates of the nodes which makes the busy periods shorter, whereas MDCF also looses in terms of channel utilization $U$ stemming from addition of new (virtual) nodes which leads to increased collision probability as shown in [35].

Example II: With the second example, we demonstrate the effectiveness of MDCF in air-time fairness for non-integer $N_{i}$. We present a simulation example with two nodes. Node 1 has a rate of 1 Mbps whereas the rate of node 2 is varied from $1 \mathrm{Mbps}$ to $3 \mathrm{Mbps}$ in $0.1 \mathrm{Mbps}$ steps. Both stations transmit $1500 \mathrm{~B}$ frames. The proposed method is compared to three cases where node 2 uses $\left\lfloor N_{2}\right\rfloor,\left\lceil N_{2}\right\rceil$, and the value $N_{2}$ rounded to the closest integer. The AF metrics obtained with each of these methods are plotted in Fig. 5. Not surprisingly, when $1 \leq N_{2}<1.5$ and $2 \leq N_{2}<2.5$, the rounded value is equal to $\left\lfloor N_{2}\right\rfloor$ and consequently the two curves closely follow each other. We have a similar case with $\left\lceil N_{2}\right\rceil$ when $1.5 \leq N_{2} \leq 2$ and $2.5 \leq N_{2} \leq 3$. All three curves deviate significantly from the ideal AF value of 1 , whereas MDCF stays very close, thus illustrating the effectiveness of the proposed switching mechanism. Note that although the data rates used in this simulation example are not standard values, the corresponding $N_{2}$ values can be encountered due to varying payloads, frame aggregation mechanisms, and service

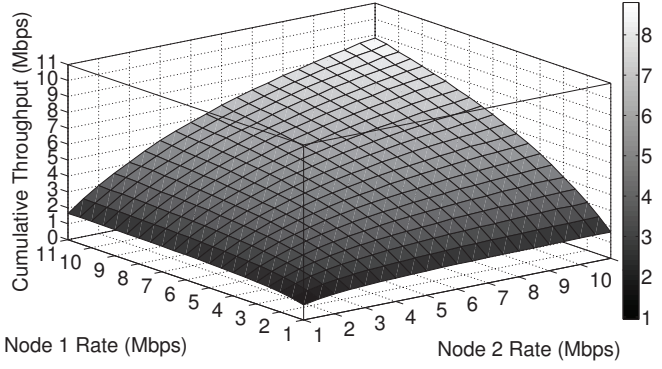

(a) Standard DCF

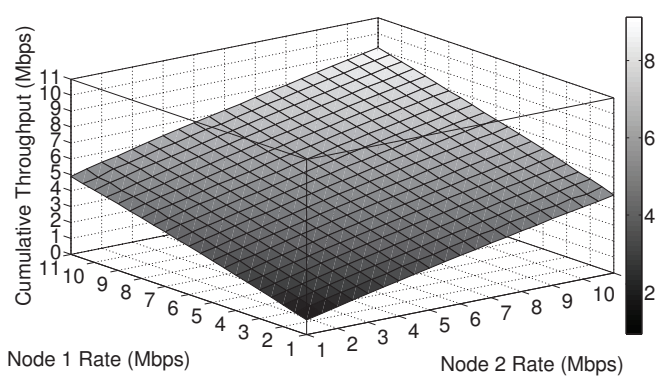

(b) MDCF

Fig. 3. Cumulative throughput comparison of standard DCF and MDCF as a function of raw bit rates.

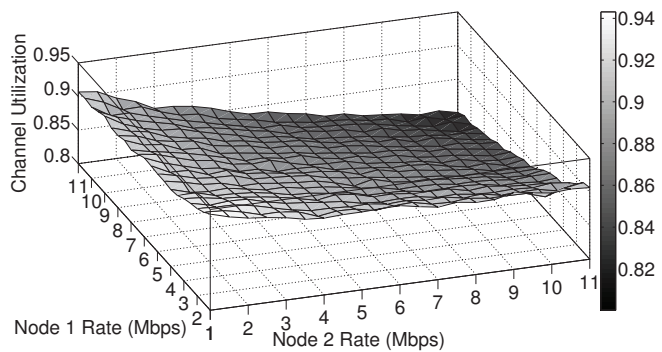

(a) Standard DCF

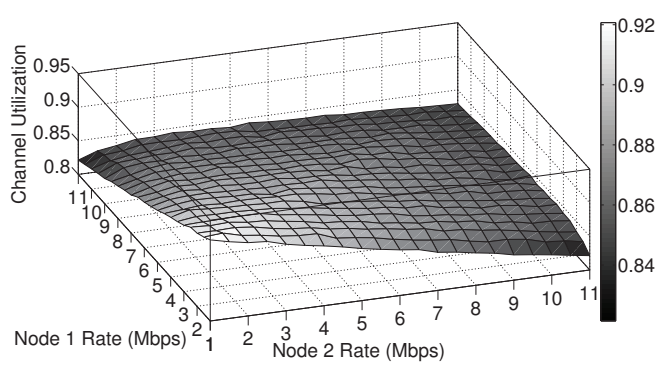

(b) MDCF

Fig. 4. Channel utilization comparison of standard DCF and MDCF as a function of raw bit rates.

differentiation schemes.

Example III: We demonstrate the scalability of MDCF in terms of the number of nodes using the system. For this purpose, we define a basic group of 4 nodes with data rates $1,2,5.5$, and $11 \mathrm{Mbps}$ and simulate scenarios with up to 10 groups of nodes. The frame length is again set to 1500 bytes for all users. Our findings that are provided in Fig. 6 show that MDCF maintains air-time fairness whereas the $A F$ parameter of the standard DCF is stuck at around 1/11 as expected. Therefore, we conclude that MDCF remains precise 


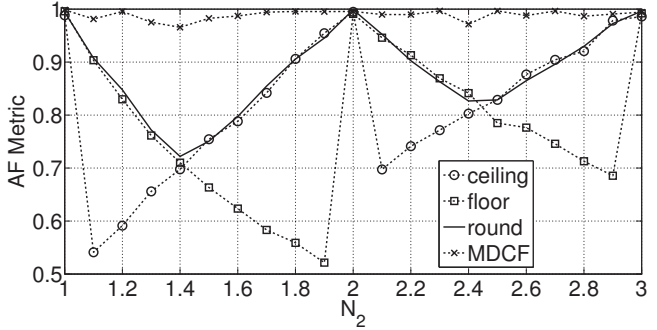

Fig. 5. AF metrics obtained with using $\left\lfloor N_{2}\right\rfloor,\left\lceil N_{2}\right\rceil$, the value $N_{2}$ rounded to the closest integer, and with the switching algorithm of MDCF. There are two stations in this scenario with $N_{1}=1$.

TABLE II

SiMULATION SCENARIO FOR EXAMPLE IV: THE DATA RATES IN MBPS AND PAYLOADS IN BYTES. U[] DENOTES THE UNIFORM DISTRIBUTION.

\begin{tabular}{|c|c|c|c|c|c|}
\hline Time (s) & St. A & St. B & St. C & St. D & St. E \\
\hline $0-100$ & \multirow{12}{*}{$\begin{array}{l}1 \mathrm{Mbps} \\
1500 \mathrm{~B}\end{array}$} & \multirow{4}{*}{$\begin{array}{l}11 \mathrm{Mbps} \\
1500 \mathrm{~B}\end{array}$} & offline & \multirow{2}{*}{ offline } & \multirow{3}{*}{ offline } \\
\hline $100-200$ & & & \multirow{4}{*}{$\begin{array}{l}5.5 \mathrm{Mbps} \\
\text { U[500,1500] }\end{array}$} & & \\
\hline $200-300$ & & & & \multirow{6}{*}{$\begin{array}{l}5.5 \mathrm{Mbps} \\
1000 \mathrm{~B}\end{array}$} & \\
\hline $300-400$ & & & & & \multirow{4}{*}{$\begin{array}{l}2 \mathrm{Mbps} \\
1000 \mathrm{~B}\end{array}$} \\
\hline $400-500$ & & \multirow{7}{*}{ offline } & & & \\
\hline $500-600$ & & & $\begin{array}{l}5.5 \mathrm{Mbps} \\
1500 \mathrm{~B}\end{array}$ & & \\
\hline $600-700$ & & & \multirow{6}{*}{$\begin{array}{l}11 \mathrm{Mbps} \\
1500 \mathrm{~B}\end{array}$} & & \\
\hline $700-800$ & & & & & \multirow{5}{*}{$\begin{array}{l}2 \mathrm{Mbps} \\
1500 \mathrm{~B}\end{array}$} \\
\hline $800-900$ & & & & $\begin{array}{l}5.5 \mathrm{Mbps} \\
\text { U[500,1500] }\end{array}$ & \\
\hline 900-1000 & & & & $\begin{array}{l}5.5 \text { Mbps } \\
\text { U[1000,1500] }\end{array}$ & \\
\hline $1000-1100$ & & & & \multirow{2}{*}{\begin{tabular}{|l}
2 Mbps \\
U[1000,1500]
\end{tabular}} & \\
\hline $1100-1200$ & & $\begin{array}{l}11 \mathrm{Mbps} \\
1500 \mathrm{~B}\end{array}$ & & & \\
\hline
\end{tabular}

even in scenarios with a relatively large number of nodes.

Example IV: With the fourth example, we demonstrate the performance of the proposed method under scenarios in which the behavior of the stations vary with time. We simulate a scenario with five stations A-E. The rates and the frame sizes of each station throughout the simulation are summarized in Table II. To clarify, Station C for instance, has no frames to send in the first 100 seconds. Then, it starts transmitting frames whose lengths are uniformly distributed between 500 and 1500 bytes, with a raw rate of $5.5 \mathrm{Mbps}$. At 500 seconds, its frame size is fixed to 1500 bytes. Another 100 seconds later, its rate becomes $11 \mathrm{Mbps}$. In addition to stations becoming online and going offline, the envisaged scenario encompasses a number of possible stress conditions including variable frame sizes and rate changes. For the payload estimates, (7) is used online with $\alpha=0.95$. The air-time utilizations of individual stations $\left(U_{i}\right)$ are plotted in Fig. 7. The $U_{i}$ curves converging to the same value following each disturbance demonstrates the effectiveness of our proposed method in transient situations as well as the steady-state. The online averaging algorithm given in (7) increases the time required for convergence when frames are variable-sized. The particular common air-time utilization value after convergence depends on the number of online stations for the corresponding scenario as well as the type of online stations.
Example V: An observation one can make due to examples I and III is that addition of new nodes to a system running MDCF slightly worsens the performance in terms of channel utilization more than it does in the case of standard DCF in general. This is due to the fact that adding a single node to an MDCF system simply means adding potentially multiple virtual nodes, leading to increased collision probability, as opposed to the addition of just one node under standard DCF. In order to mitigate this adverse effect, we propose using frame aggregation in conjunction with MDCF. We let each node aggregate a number of frames into a burst at each transmission attempt in a way that its air-time requirement for the burst does not exceed $A_{\max }$ (the air-time required for the slowest node to transmit one single frame), and the number of frames aggregated does not exceed a predetermined value denoted by $F_{\max }$. In this example, we demonstrate the performance of MDCF with the described frame aggregation scheme. We simulate MDCF with four nodes with rates $1,2,5.5$, and 11 Mbps, all using frame sizes of 1500 bytes while varying the parameter $F_{\max }$. For MDCF, we use two $C W_{\min }$ values, 156 and 128 , the latter being an integer power of two. We let $F_{a g g}$ denote the number of aggregated frames. The results are given in Table III. When we are allowed to aggregate a few frames, i.e., $F_{\max }>1$, the number of virtual nodes used for MDCF per node is reduced thus increasing the channel utilization with respect to the case $F_{\max }=1$. We conclude that MDCF with moderate frame aggregation, i.e., $F_{\max }=3$, meets the airtime fairness requirement with a channel utilization surpassing that of the standard DCF. Consequently, there may not be any need for aggressive frame aggregation policies such as the one with $F_{\max }=11$ recalling that such policies may lead to short-term unfairness among nodes. Moreover, the results of $C W_{\min }$ being 156 or 128 are only slightly different and $C W_{\min }$ can also be chosen to be a power of two in MDCF in real implementations without compromising from air-time fairness. In this example, we assume that back-to-back frames are sent without any gaps within between but more general frame aggregation schemes including the ones mentioned in [27] can also be used.

Example VI: In the final example, we compare the performance of MDCF to the technique of $C W_{\min }$ adjustment in the context service differentiation. In this scenario, which has been inspired from [15] (see Fig. 2), all stations have 11 Mbps rates and their frame sizes are 1500 bytes. There are two classes into which the stations are evenly distributed. The stations in the high priority class are to be given twice the throughput that the stations in the low priority class. For this purpose, in the $C W_{\min }$ adjustment technique, the low priority stations are assigned a $C W_{\min }$ value that is twice that of the high priority stations. On the other hand, with MDCF, high priority stations run two back-off algorithms $(N=2)$ each, as opposed to a single algorithm run by each low priority station.

In Fig. 8, the ratios of the throughput obtained by the high priority class to that of the low priority class is plotted as a function of the total number of stations in the system for various $C W_{\min }$ values. In the graph for the $C W_{\min }$ adjustment technique, the $C W_{\min }$ values given in the legend are used by high priority nodes, whereas the $C W_{\min }$ values are the same for all stations in MDCF and are given in the corresponding 

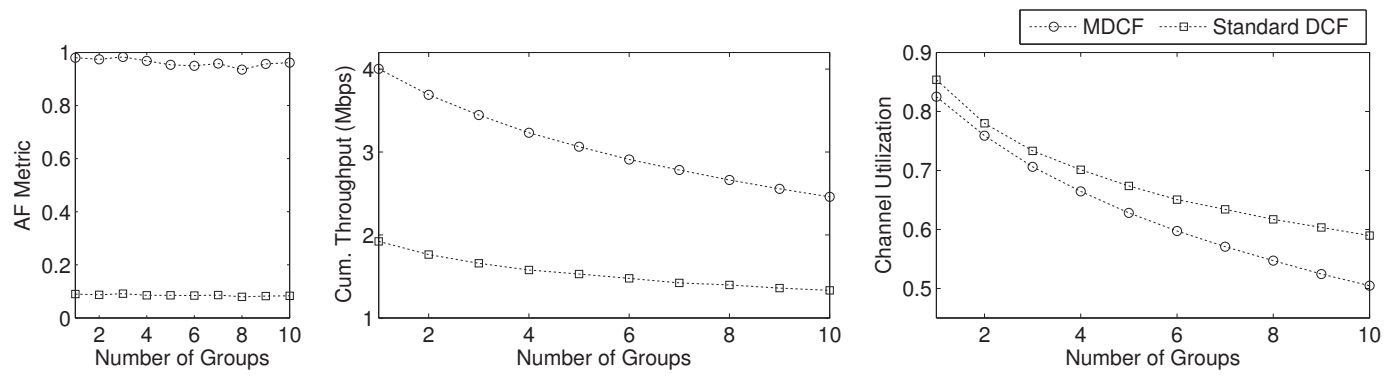

Fig. 6. Comparison of standard DCF and MDCF under scenarios with up to 40 nodes.

TABLE III

MDCF S YSTEM PERFORMANCE METRICS $A F, T$, AND $U$, AS A FUNCTION OF THE FRAME AGGREGATION PARAMETER $F_{m a x}$ FOR TWO VALUES OF $C W_{\min }^{(M D C F)}$ FOR A SCENARIO WITH FOUR NODES WITH FOUR DIFFERENT RAW BIT RATES.

\begin{tabular}{|c|c|c|c|c|c|c|c|c|c|c|c|c|c|c|}
\hline \multirow{3}{*}{$\frac{\text { Node: }}{F_{\max }}$} & \multicolumn{2}{|c|}{$1 \mathrm{Mbps}$} & \multicolumn{2}{|c|}{2 Mbps } & \multicolumn{2}{|c|}{ 5.5 Mbps } & \multicolumn{2}{|c|}{$11 \mathrm{Mbps}$} & \multicolumn{2}{|c|}{$A F$} & \multicolumn{2}{|c|}{$T$ (Mbps) } & \multicolumn{2}{|c|}{$U$} \\
\hline & $F_{a g g}$ & $N$ & $F_{a g g}$ & $N$ & $F_{a g g}$ & $N$ & $F_{a g g}$ & $N$ & \multicolumn{2}{|c|}{$C W_{\min }^{(M D C F)}$} & \multicolumn{2}{|c|}{$C W_{\min }^{(M D C F)}$} & \multicolumn{2}{|c|}{$C W_{\min }^{(M D C F)}$} \\
\hline & & & & & & & & & & n 128 & & 128 & $156^{n}$ & n 128 \\
\hline 1 & 1 & 1 & 1 & 2 & 1 & 5.5 & 1 & 11 & 0.9826 & 0.9774 & 4.011 & 3.952 & 0.8243 & 0.8136 \\
\hline 2 & 1 & 1 & 2 & 1 & 2 & 2.75 & 2 & 5.5 & 0.9959 & 0.9859 & 4.313 & 4.285 & 0.8856 & 0.8805 \\
\hline 3 & 1 & 1 & 2 & 1 & 3 & 1.833 & 3 & 3.667 & 0.9653 & 0.9843 & 4.453 & 4.397 & 0.9086 & 0.9061 \\
\hline 4 & 1 & 1 & 2 & 1 & 4 & 1.375 & 4 & 2.75 & 0.9851 & 0.9798 & 4.484 & 4.471 & 0.9210 & 0.9202 \\
\hline 5 & 1 & 1 & 2 & 1 & 5 & 1.1 & 5 & 2.2 & 0.9671 & 0.9667 & 4.501 & 4.530 & 0.9282 & 0.9276 \\
\hline 6 & 1 & 1 & 2 & 1 & 5 & 1.1 & 6 & 1.833 & 0.9892 & 0.9914 & 4.546 & 4.533 & 0.9335 & 0.9318 \\
\hline 7 & 1 & 1 & 2 & 1 & 5 & 1.1 & 7 & 1.571 & 0.9744 & 0.9681 & 4.535 & 4.526 & 0.9340 & 0.9349 \\
\hline 8 & 1 & 1 & 2 & 1 & 5 & 1.1 & 8 & 1.375 & 0.9515 & 0.9742 & 4.513 & 4.616 & 0.9376 & 0.9392 \\
\hline 9 & 1 & 1 & 2 & 1 & 5 & 1.1 & 9 & 1.222 & 0.9649 & 0.9688 & 4.599 & 4.636 & 0.9370 & 0.9414 \\
\hline 10 & 1 & 1 & 2 & 1 & 5 & 1.1 & 10 & 1.1 & 0.9710 & 0.9615 & 4.587 & 4.632 & 0.9401 & 0.9408 \\
\hline 11 & 1 & 1 & 2 & 1 & 5 & 1.1 & 11 & 1 & 0.9849 & 0.9835 & 4.579 & 4.593 & 0.9406 & 0.9412 \\
\hline
\end{tabular}

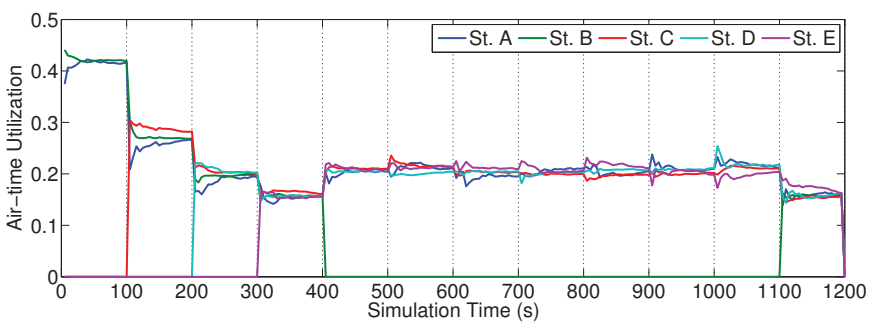

Fig. 7. Air-time utilization for each station under the scenario summarized in Table II.

legend. As the number of overall stations increases, $C W_{\min }$ adjustment technique has difficulty maintaining the required throughput ratio especially with lower $C W_{\min }$ values, dropping to as low as 1.75 whereas the ratios obtained by MDCF remains within the range $[1.973,2.025]$. We plot the channel utilization under this scenario in Fig. 9, which shows similarity between the performances of $C W_{\text {min }}$ adjustment and MDCF. On the other hand, both figures indicate that the performance of $C W_{\min }$ adjustment is sensitive to the specific scenario it is run. Bearing in mind that we aim to come up with a distributed scheme, it is likely that a predetermined and fixed set of parameters will be used under any scenario. This leads us to believe that one can not expect a consistent level of throughput differentiation from $C W_{\min }$ adjustment, contrary to what appears to be valid for MDCF.

\section{CONCLUSION}

We presented a distributed air-time fair MAC (MDCF) for multi-rate WLANs by allowing multiple instances of the DCF back-off algorithm to be run at each node. We also propose
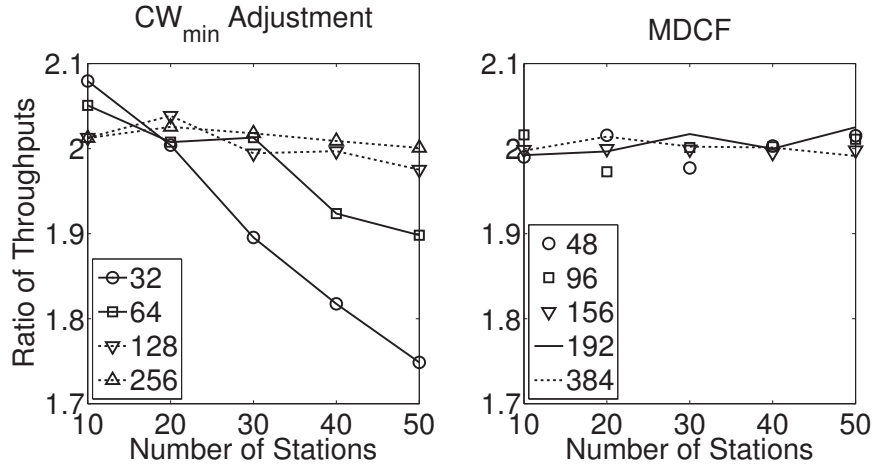

Fig. 8. Ratios of the throughput obtained by the high priority class to that of the low priority class for various network sizes and $C W_{\min }$ values.

to use MDCF together with frame aggregation to alleviate the slight reduction in channel utilization observed for MDCF without frame aggregation. We show that MDCF achieves air-time fairness in all the scenarios we studied. Although MDCF is proposed as an instrument for air-time fairness in the current paper, it can also be used for service differentiation in WLANs as shown in Example VI, which is a topic for further exploration. The impact of non-zero wireless packet errors and the capture effect on MDCF performance is also left for future research.

\section{APPENDIX A}

\section{ProOF OF AIR-TIME FAIRNESS OF MDCF FOR NON-INTEGER $N_{i}$}

For the sake of convenience, let $K=2$ first. Note that user 2 now alternates between $N_{2-}$ and $N_{2+}$ instances of 

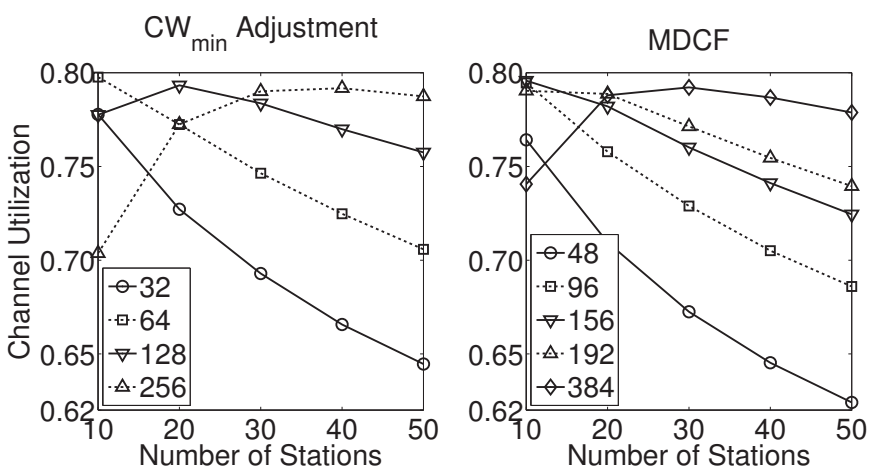

Fig. 9. Channel utilization for various network sizes and $C W_{\min }$ values.

the DCF according to the switching parameter $a_{2}$ as defined in (6). Let $X_{i}(k)$ denote the number of instances of DCF run by user $i$ just before the $k$ th transmission in the system. Then the process $X(k)=\left\{\left(X_{1}(k), X_{2}(k)\right): k \geq 1\right)$ is a discrete-time Markov chain with four states, namely the states $\left(N_{1-}, N_{2-}\right),\left(N_{1-}, N_{2+}\right),\left(N_{1+}, N_{2-}\right)$, and $\left(N_{1+}, N_{2+}\right)$. The transition diagram of this Markov chain is depicted in Fig. 10.

Let $\pi(k, l), k=N_{1-}, N_{1+}, l=N_{2-}, N_{2+}$ denote the steady-state distribution of this Markov chain. It is not difficult to show that this Markov chain is reversible and its steadystate distribution is explicitly given by:

$\pi\left(N_{1-}, N_{2-}\right)=\frac{\left(N_{1+}-N_{1}\right)\left(N_{2+}-N_{2}\right)\left(N_{1-}+N_{2-}\right)}{\left(N_{1}+N_{2}\right)}$,
$\pi\left(N_{1-}, N_{2+}\right)=\frac{\left(N_{1+}-N_{1}\right)\left(N_{2}-N_{2-}\right)\left(N_{1-}+N_{2+}\right)}{\left(N_{1}+N_{2}\right)}$,
$\pi\left(N_{1+}, N_{2-}\right)=\frac{\left(N_{1}-N_{1-}\right)\left(N_{2+}-N_{2}\right)\left(N_{1+}+N_{2-}\right)}{\left(N_{1}+N_{2}\right)}$,
$\pi\left(N_{1+}, N_{2+}\right)=\frac{\left(N_{1}-N_{1-}\right)\left(N_{2}-N_{2-}\right)\left(N_{1+}+N_{2+}\right)}{\left(N_{1}+N_{2}\right)}$.

To show this, one can substitute the above distribution and verify the detailed balance equations. A packet is transmitted by user 1 at state $\left(N_{1-}, N_{2-}\right)$ with probability $N_{1-} /\left(N_{1-}+\right.$ $\left.N_{2-}\right)$ or at state $\left(N_{1-}, N_{2+}\right)$ with probability $N_{1-} /\left(N_{1-}+\right.$ $\left.N_{2+}\right)$ and so on. Therefore,

$p_{1}=\sum_{k=N_{1-}, N_{1+}} \sum_{l=N_{2-}, N_{2+}} \pi(k, l) k /(k+l)=N_{1} /\left(N_{1}+N_{2}\right)$.

Similarly, $p_{2}=N_{2} /\left(N_{1}+N_{2}\right)$. One can also show by similar algebra that $p_{i}=N_{i} / \sum_{j=1}^{K} N_{j}$ for the more general case $K>2$ by making use of the reversibility of the underlying Markov chain but the proof is more elaborate and is left out deliberately. Therefore, we conclude that the expression for $p_{i}^{(M D C F)}$ in (3) remains intact for non-integer $N_{i}$.

\section{ACKNOWLEDGMENT}

This work is supported in part by TÜBİTAK (The Scientific and Technological Research Council of Turkey) grant no. $111 \mathrm{E} 106$.

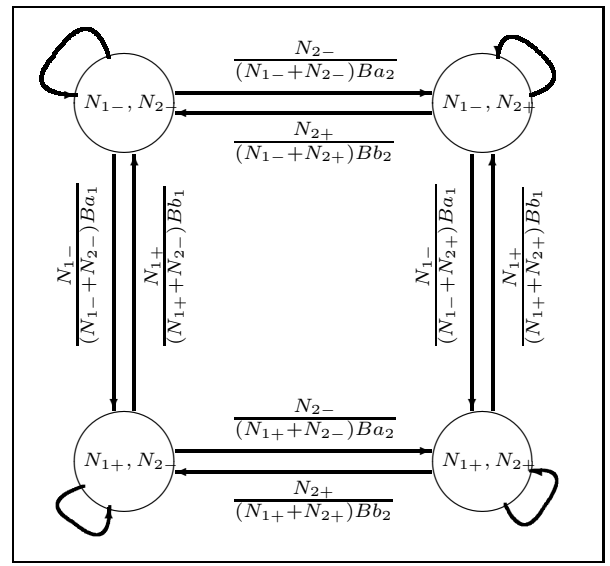

Fig. 10. The transition diagram of the four-state Markov chain described for the two-user scenario.

\section{REFERENCES}

[1] ANSI/IEEE, 802.11: Wireless LAN Medium Access Protocol (MAC) and Physical Layer (PHY) Specifications, IEEE, 2007.

[2] _ , 802.11a: Wireless LAN Medium Access Protocol (MAC) and Physical Layer (PHY) Specifications, IEEE, 1999.

[3] — , 802.11g: Wireless LAN Medium Access Protocol (MAC) and Physical Layer (PHY) Specifications, IEEE, 2003.

[4] Y. Xiao and J. Rosdahl, "Throughput and delay limits of IEEE 802.11," IEEE Commun. Lett., vol. 6, no. 8, pp. 355-357, 2002.

[5] T. Paul and T. Ogunfunmi, "Wireless LAN comes of age: understanding the IEEE 802.11n amendment," IEEE Circuits and Systems Mag., vol. 8, no. 1, pp. 28-54, 2008.

[6] D. Skordoulis, Q. Ni, H.-H. Chen, A. Stephens, C. Liu, and A. Jamalipour, "IEEE 802.11n MAC frame aggregation mechanisms for nextgeneration high-throughput WLANs," IEEE Wireless Commun., vol. 15, no. 1 , pp. 40-47, 2008.

[7] ANSI/IEEE, 802.11b: Wireless LAN Medium Access Protocol (MAC) and Physical Layer (PHY) Specifications, IEEE, 1999.

[8] D. Qiao, S. Choi, and K. Shin, "Goodput analysis and link adaptation for IEEE 802.11a wireless LANs," IEEE Trans. Mobile Comput., vol. 1, no. 4, pp. 278-292, Oct. 2002.

[9] M. Heusse, F. Rousseau, G. Berger-Sabbatel, and A. Duda, "Performance anomaly of $802.11 \mathrm{~b}$," in Proc. 2003 IEEE INFOCOM.

[10] G. Tan and J. Guttag, "Long-term time-share guarantees are necessary for wireless LANs," in in Proc. 2004 SIGOPS European Workshop.

[11] —, "Time-based fairness improves performance in multi-rate WLANs," in 2004 USENIX.

[12] A. Checco and D. Leith, "Proportional fairness in 802.11 wireless LANs," IEEE Commun. Lett., vol. 15, no. 8, pp. 807-809, 2011.

[13] B. Li and R. Battiti, "Performance analysis of an enhanced IEEE 802.11 distributed coordination function supporting service differentiation," in Proc. 2003 Quality for All, 4th COST 263 International Workshop on Quality of Future, Internet Services, ser. Lecture Notes in Computer Science, G. Karlsson and M. I. Smirnov, Eds., vol. 2811. Springer, 2003, pp. 152-161.

[14] H. Kim, S. Yun, I. Kang, and S. Bahk, "Resolving 802.11 performance anomalies through QoS differentiation," IEEE Commun. Lett., vol. 9, pp. 555-557, July 2005.

[15] I. Tinnirello, G. Bianchi, and L. Scalia, "Performance evaluation of differentiated access mechanisms effectiveness in 802.11 networks," in Proc. 2004 IEEE Global Telecommunications Conference, vol. 5, pp. 3007-3011.

[16] V. A. Siris and G. Stamatakis, "Optimal CWmin selection for achieving proportional fairness in multi-rate 802.11e WLANs: test-bed implementation and evaluation," in Proc. 2006 International Workshop on Wireless Network Testbeds, Experimental Evaluation \& Characterization, ser. WiNTECH '06. ACM, 2006, pp. 41-48.

[17] Y. Chetoui and N. Bouabdallah, "Adjustment mechanism for the IEEE 802.11 contention window: An efficient bandwidth sharing scheme," Computer Commun., vol. 30, no. 13, pp. 2686-2695, Sept. 2007.

[18] C.-T. Chou, K. Shin, and N. Sai Shankar, "Contention-based airtime usage control in multirate IEEE 802.11 wireless LANs," IEEE/ACM Trans. Networking, vol. 14, no. 6, pp. 1179-1192, 2006. 
[19] P. Lin, W. i Chou, and T. Lin, "Achieving airtime fairness of delaysensitive applications in multirate ieee 802.11 wireless LANs," IEEE Commun. Mag., vol. 49, no. 9, pp. 169-175, 2011.

[20] T. Joshi, A. Mukherjee, Y. Yoo, and D. P. Agrawal, "Airtime fairness for IEEE 802.11 multirate networks," IEEE Trans. Mobile Comput., vol. 7, pp. 513-527, Apr. 2008.

[21] M. Heusse, F. Rousseau, R. Guillier, and A. Duda, "Idle Sense: an optimal access method for high throughput and fairness in rate diverse wireless LANs," 2005 SIGCOMM.

[22] L. Iannone and S. Fdida, "Un schema a division detemps pour eviter lanomalie de la couche MAC 802.11b," in 2005 Colloque Francophone sur IIngenierie des Protocoles.

[23] J. Dunn, M. Neufeld, A. Sheth, D. Grunwald, and J. Bennett, "A practical cross-layer mechanism for fairness in 802.11 networks," Mob. Netw. Appl., vol. 11, no. 1, pp. 37-45, 2006.

[24] T. Razafindralambo, I. G. Lassous, L. Iannone, and S. Fdida, "Dynamic packet aggregation to solve performance anomaly in 802.11 wireless networks," in Proc. 2006 ACM International Symposium on Modeling Analysis and Simulation of Wireless and Mobile Systems, pp. 247-254.

[25] Y. Lin and V. Wong, "Frame aggregation and optimal frame size adaptation for IEEE 802.11n WLANs," in 2006 IEEE Global Telecommunications Conference, Nov. 2006.

[26] M. Ergen and P. Varaiya, "Formulation of distributed coordination function of IEEE 802.11 for asynchronous networks: mixed data rate and packet size," IEEE Trans. Veh. Technol., vol. 57, no. 1, pp. 436-447, 2008.

[27] I. Tinnirello and S. Choi, "Temporal fairness provisioning in multi-rate contention-based 802.11e WLANs," in Proc. 2005 IEEE International Symposium on World of Wireless Mobile and Multimedia Networks, pp. 220-230.

[28] I. Inan, F. Keceli, and E. Ayanoglu, "Analysis of the 802.11e enhanced distributed channel access function," IEEE Trans. Commun., vol. 57, no. 6, pp. 1753-1764, 2009.

[29] K. Kashibuchi, Y. Nemoto, and N. Kato, "Mitigating performance anomaly of TFRC in multi-rate IEEE 802.11 wireless LANs," in Proc. 2009 IEEE GLOBECOM.

[30] K. Kashibuchi, T. Taleb, Y. Nemoto, and N. Kato, "Channel occupancy time based TCP rate control for IEEE 802.11 DCF," 2008 IWCMC.

[31] C.-W. Huang, M. Loiacono, J. Rosca, and J.-N. Hwang, "Airtime fair distributed cross-layer congestion control for real-time video over WLAN," IEEE Trans. Circuits and Systems for Video Technol., vol. 19, no. 8, pp. 1158-1168, 2009.

[32] F. Keceli, I. Inan, and E. Ayanoglu, "Weighted fair uplink/downlink access provisioning in IEEE 802.11e WLANs," in Proc. 2008 IEEE ICC, pp. 2473-2479.

[33] W.-S. Lim, D.-W. Kim, and Y.-J. Suh, "Achieving fairness between uplink and downlink flows in error-prone WLANs," IEEE Commun. Lett., vol. 15, no. 8, pp. 822-824, Aug. 2011.

[34] C. Wang, H.-K. Lo, and S.-H. Fang, "Fairness analysis of throughput and delay in WLAN environments with channel diversities," EURASIP J. Wireless Commun. and Networking, 2011.

[35] G. Bianchi, "Performance analysis of the IEEE 802.11 distributed coordination function," IEEE J. Sel. Areas Commun., vol. 18, no. 3, pp. 533-547, 2000.

[36] E. Elhag and M. Othman, "Adaptive contention window scheme for WLANs," International Arab J. Inf. Technol., vol. 4, no. 4, pp. 313 321, Oct. 2007.

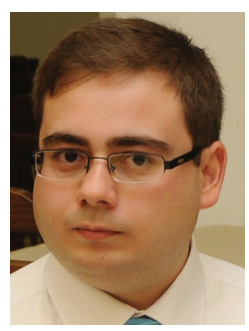

Mehmet Akif Yazici earned his B.S. and M.S. degrees in Electrical and Electronics Engineering from Middle East Technical University, Turkey, in 2004 and 2006, respectively. He is currently a Ph.D. candidate in the Department of Electrical and Electronics Engineering, Bilkent University, Turkey. His research interests include computer networks, with emphasis on stochastic modeling, and analysis of telecommunication systems and networks.

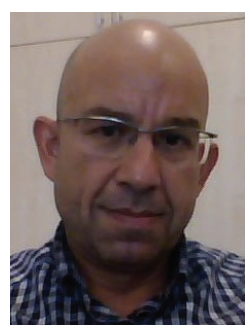

Nail Akar received his B.S. degree from Middle East Technical University, Turkey, in 1987, and his M.S. and Ph.D. degrees from Bilkent University, Ankara, Turkey, in 1989 and 1994, respectively, all in Electrical and Electronics Engineering. From 1994 to 1996 , he was a visiting scholar and a visiting assistant professor in the Computer Science Telecommunications program at the University of Missouri-Kansas City. He joined the Technology Planning and Integration group at Long Distance Division, Sprint, Overland Park, Kansas, in 1996, where he held a senior member of technical staff position from 1999 to 2000 . Since 2000, he has been with Bilkent University, Turkey, currently as an associate professor in the Electrical and Electronics Engineering Department. His current research interests include performance analysis of computer and communication systems and networks, performance evaluation tools and methodologies, design and engineering of optical and wireless networks, queuing systems, and resource management. 